ARTICLE

https://doi.org/10.1038/s41467-019-12278-3

\title{
Descent trajectory reconstruction and landing site positioning of Chang'E-4 on the lunar farside
}

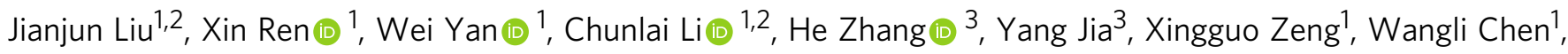

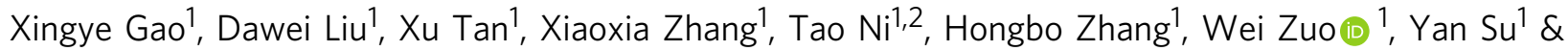
Weibin Wen ${ }^{1}$

Chang'E-4 (CE-4) was the first mission to accomplish the goal of a successful soft landing on the lunar farside. The landing trajectory and the location of the landing site can be effectively reconstructed and determined using series of images obtained during descent when there were no Earth-based radio tracking and the telemetry data. Here we reconstructed the powered descent trajectory of CE-4 using photogrammetrically processed images of the CE-4 landing camera, navigation camera, and terrain data of Chang'E-2. We confirmed that the precise location of the landing site is $177.5991^{\circ} \mathrm{E}, 45.4446^{\circ} \mathrm{S}$ with an elevation of $-5935 \mathrm{~m}$. The landing location was accurately identified with lunar imagery and terrain data with spatial resolutions of $7 \mathrm{~m} / \mathrm{p}, 5 \mathrm{~m} / \mathrm{p}, 1 \mathrm{~m} / \mathrm{p}, 10 \mathrm{~cm} / \mathrm{p}$ and $5 \mathrm{~cm} / \mathrm{p}$. These results will provide geodetic data for the study of lunar control points, high-precision lunar mapping, and subsequent lunar exploration, such as by the Yutu-2 rover.

\footnotetext{
${ }^{1}$ Key Laboratory of Lunar and Deep Space Exploration, National Astronomical Observatories, Chinese Academy of Sciences, Beijing 100101, China. ${ }^{2}$ School of Astronomy and Space Science, University of Chinese Academy of Sciences, Beijing 100049, China. ${ }^{3}$ China Academy of Space Technology, Beijing 100094 , China. Correspondence and requests for materials should be addressed to C.L. (email: licl@nao.cas.cn)
} 
$\mathrm{T}$ he Chang'E-4 (CE-4) spacecraft successfully landed on the lunar farside on January 3, 2019. The planned durations of the scientific investigations for the lander and the Yutu-2 rover are 6 and 3 months, respectively. After launch on December 8,2018 , CE-4 conducted several stages, including transfer from the Earth to the Moon, orbiting around the Moon and a powered descent. The powered descent stage was the most important stage for soft landing and was implemented under complete autonomous control ${ }^{1}$. According to the orbit design, a 7500 Newton variable propulsion engine was used for deceleration during the CE-4 powered descent stage, which involved phases of main braking, attitude adjusting, vertical descent, hover and hazardavoidance, slow descent ${ }^{1-5}$. To better understand the autonomous control result and to service orbit control strategy analysis and detection mission planning for subsequent missions, it is of great significance to accurately reconstruct the landing trajectory and determine the location of the landing site after a safe landing.

Radio measurements $s^{6-12}$, real-time telemetry data $^{2,13}$, and image-based matching ${ }^{3,14-19}$ are commonly used for powered descent trajectory reconstruction and landing site positioning on a planetary surface. Because there was no radio measurement equipment between the CE-4 and the relay satellite Queqiao, it was unable to perform direct or indirect radio measurements by the ground tracking network on the lunar farside. In addition, the telemetry data received by Queqiao (including the detector altitude, acceleration, and attitude) were not released and could not to be used. As a result, it is difficult to accurately reconstruct the spacecraft's landing trajectory and to confirm the precise landing site location. However, these problems can be effectively solved through localization technology based on landing images, which is not affected by factors such as the lunar gravity field and the dynamical model.

In this study, high-frequency landing sequence images transmitted by Queqiao after safe landing were used for descent trajectory reconstruction and landing site positioning of CE-4, which completely recorded the entire process of the powered descent. We reconstructed the descent trajectory, showing even barely perceivable maneuvers of the spacecraft during the landing approach and that the landing site can also be precisely localized. Here, the digital orthophoto map (DOM) and digital elevation model (DEM) of Chang'E-2 (CE2TMAP2015, see the data descriptions of the Methods section ${ }^{20,21}$ ) were used as the geographical reference data. The CE-4 landing camera (LCAM, see the Methods section for details) sequence images were applied to reconstruct the CE-4 powered descent trajectory by the photogrammetry bundle adjustment technology ${ }^{22,23}$ (see the Methods for a detailed technical flow). Using combined binocular stereoscopic images obtained by the navigation camera (NCAM, see the Methods), which consists of two cameras, we confirmed that the precise location of the landing site is $177.5991^{\circ} \mathrm{E}, 45.4446^{\circ} \mathrm{S}$ with an elevation of $-5935 \mathrm{~m}$.

\section{Results}

Descent trajectory from LCAM images. The sequence images obtained by the LCAM in vertical attitude after the attitude adjusting phase of CE- 4 were used for the reconstruction of the landing trajectory in this study.

According to the reconstructed trajectory, CE-4 adopted a vertical descent approach to gradually approach the landing zone after the attitude adjusting phase with an altitude of $5635 \mathrm{~m}$ and a velocity of $\sim 85 \mathrm{~m} / \mathrm{s}$. During the period of descending to an altitude of $4130 \mathrm{~m}$ above the lunar surface, the trajectory moved $77 \mathrm{~m}$ to the north and crossed a crater with a diameter of approximately $200 \mathrm{~m}$. CE-4 reached over area A, as indicated in Fig. 1, with a velocity of $\sim 78 \mathrm{~m} / \mathrm{s}$. Over area A, CE-4 decreased from $4130 \mathrm{~m}$ from the lunar surface to $1495 \mathrm{~m}$, the velocity decreased to $\sim 51 \mathrm{~m} / \mathrm{s}$, and the trajectory was adjusted from the northeast; there are some craters with diameters of $~ 70-100 \mathrm{~m}$ to the northwest, where is more flat (Fig. 1d, e). Then, the trajectory moved $244 \mathrm{~m}$ to the northwest from the altitude of $1495 \mathrm{~m}$ to $99 \mathrm{~m}$ and the velocity decreased to $0 \mathrm{~m} / \mathrm{s}$. CE-4 reached over area $B$ in Fig. 1 and entered the hover and hazard-avoidance phase.

At an altitude of $99 \mathrm{~m}$ above the lunar surface (B in Fig. 1), CE4 hovered for $\sim 13 \mathrm{~s}$. Then, the trajectory moved $12 \mathrm{~m}$ to the southwest and crossed the crater with a diameter of $25 \mathrm{~m}$. The altitude above the lunar surface decreased to $30 \mathrm{~m}$, and the velocity changed to $\sim 1.5 \mathrm{~m} / \mathrm{s}$. Finally, the CE- 4 trajectory slowly descended vertically and, the spacecraft landed safely.

Landing site localization based on multisource image data. We generated LCAM topographic maps (including the DOM and DEM data) with different spatial resolutions by photogrammetry bundle adjustment technology 22,23 . These maps used a total of 180 images from the LCAM sequence images below an altitude of $5635 \mathrm{~m}$ (one image per second), twelve horizontal and vertical control points (GCPs), and one vertical GCP selected on the CE2TMap2015 (Fig. 2a). Using the stereoscopic images obtained by the NCAM from the Yutu-2 rover on the top of the lander and a total of 14 GCPs selected from the LCAM DOM and DEM (Fig. 2b), the DOM with a resolution of $2 \mathrm{~cm} / \mathrm{p}$ was produced by the close-range photogrammetry technology. In this way, the NCAM DOM was precisely registered to the LCAM DOM. According to the position relationship between the NCAM and the origin of the lander body coordinate system, the horizontal position and elevation of the lander in the LCAM terrain data were measured, which were determined as the final position of the CE-4 landing site (see the Methods). Detailed information on the location of the landing site is shown in Table 1 and Fig. 3.

We also marked the location of the landing site on the LCAM images and CE-2 DOM subdivision with a resolution of $7 \mathrm{~m} / \mathrm{p}$ (Table 1 and Fig. 4).

The images of the LCAM and Yutu-2 panoramic camera (PCAM) (Fig. 5) show that CE-4 landed on a gentle slope of a degraded crater, only $8.35 \mathrm{~m}$ from the rim of a $25 \mathrm{~m}$ crater to the north and was surrounded by 5 craters with diameters of $10.21 \sim 25.00 \mathrm{~m}$ and depths of 1.21 3.03 m. CE-4 landing site can be also located in the Lunar Reconnaissance Orbiter (LRO) narrow angle camera (NAC) images, whose relative position to the surrounding terrain is consistent with our results in Fig. 5.

\section{Discussion}

Similar to Chang'E-3 (CE-3), three types of sensors were employed by CE-4, including a laser ranging sensor, a microwave ranging and velocimeter sensor and an optical imaging sensor and a laser imaging sensor ${ }^{1}$. The powered descent process was realized by autonomous control technology with adaptive powered explicit guidance, multibeam fault-tolerant navigation, and partition quaternion control. However, the trace control strategies for CE-3 and CE-4 are different, and this difference is also reflected in the trajectory recovered in this article.

The CE-3 landing site is located on the northern Mare Imbrium of the lunar nearside. The length of the CE-3 nadir flight trace is $\sim 450 \mathrm{~km}$ from south to north. The terrain along the nadir flight trace shows a flat, small variation, gradually decreasing from south to north with a maximum variation of $\sim 800 \mathrm{~m}$ (Fig. 6). In the main braking phase, ranging measurements were introduced to correct the bias and drift of the inertial measurement unit (IMU) in time and ensure the stability of the powered descent trajectory. At an altitude of $3 \sim 2.4 \mathrm{~km}$, the CE-3 lander performed quick attitude adjustments to a vertical attitude. 
a

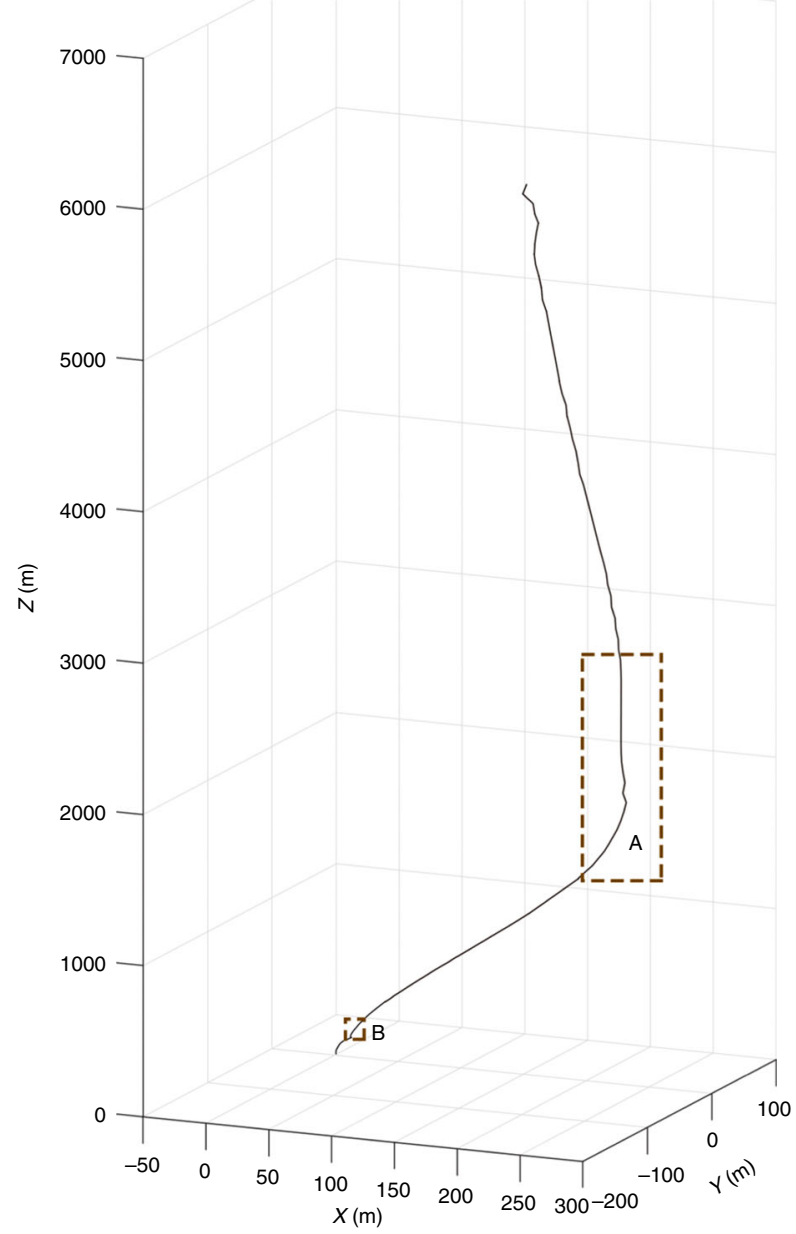

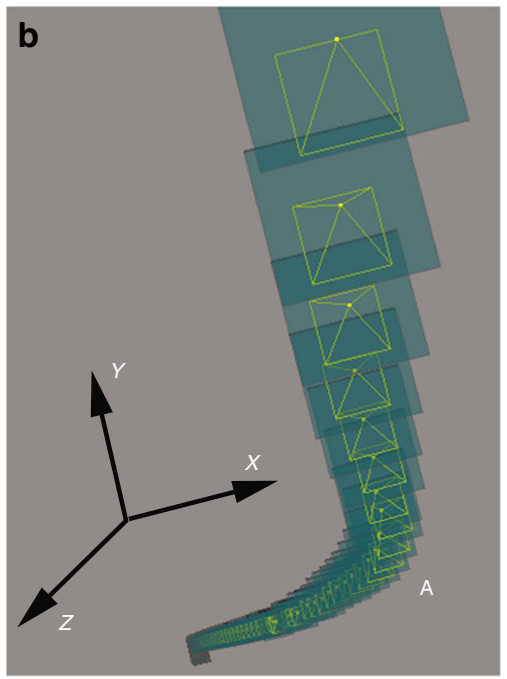

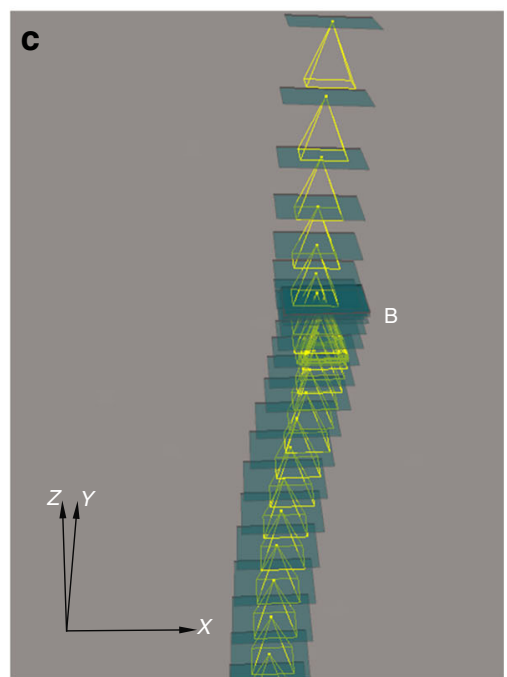

d

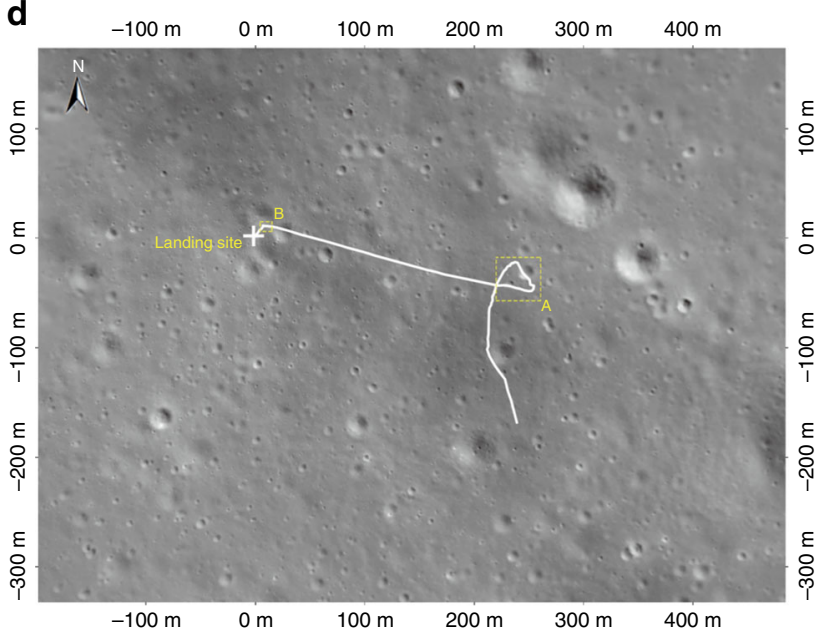

e

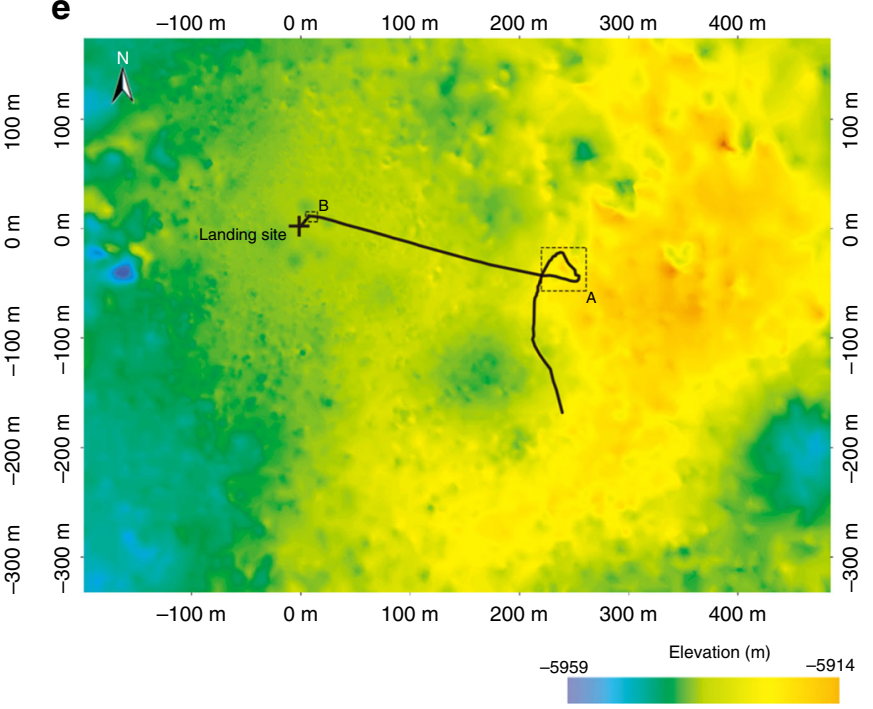

Fig. 1 CE-4 powered descent trajectory. The + is the identified landing location. a CE-4 descent trajectory from the altitude of $6000 \mathrm{~m}$ to the lunar surface; b, $\mathbf{c}$ are zoomed images of the descent trajectories of $A$ and $B$, respectively. The green box represents the position of the LCAM focal plane, and the yellow cone represents the field of view of the LCAM. $\mathbf{d}$ The solid white line is the projection of the descent trajectory on the LCAM DOM. $\mathbf{e}$ The solid black line is the projection of the descent trajectory on the LCAM DEM. The coordinate system in this figure is the tangent plane coordinate system of the landing site. This system uses the location of the landing site as the coordinate origin, with its $X$ and $Y$ axes pointing towards the geographical east and the north of the landing site, respectively. The $\mathrm{Z}$-axis, $\mathrm{X}$-axis, and $\mathrm{Y}$-axis form a right-hand system, with the $\mathrm{Z}$-axis pointing to the zenith direction of the landing site 
a

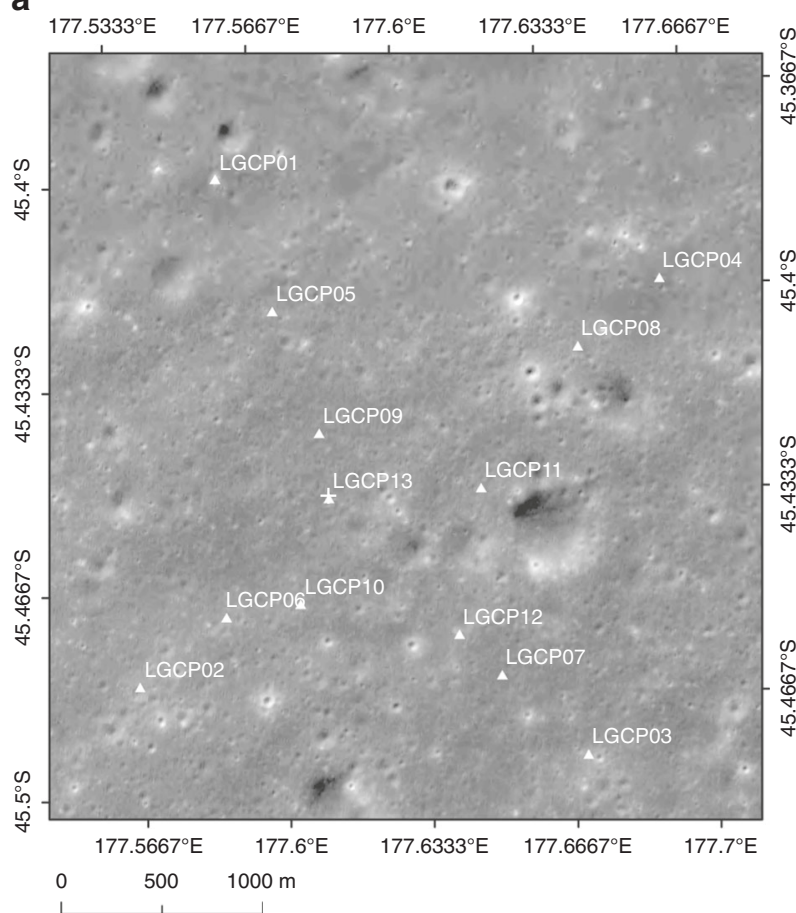

b

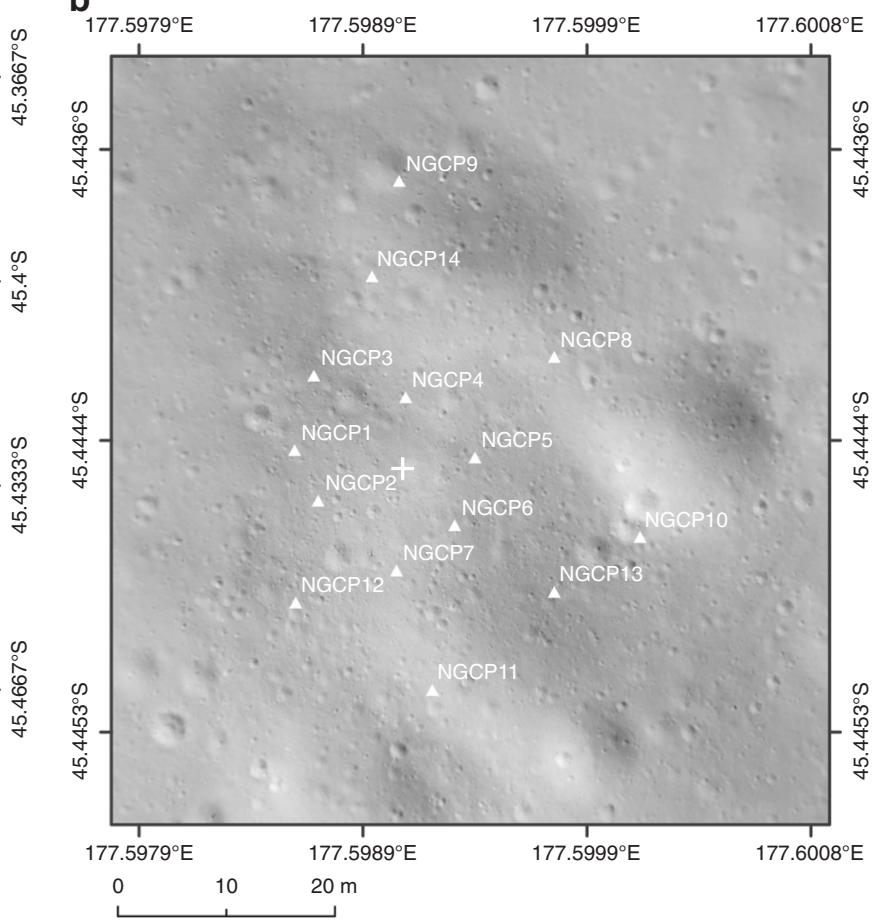

Fig. 2 GCPs map for photogrammetric adjustment processing. a GCPs on the CE2TMap2015 DOM for the LCAM image adjustment, marked as LGCPs, in which LGCP13 is the vertical GCP; b GCPs on the LCAM DOM for the NCAM image adjustment, marked as NGCPs. The + is the landing site

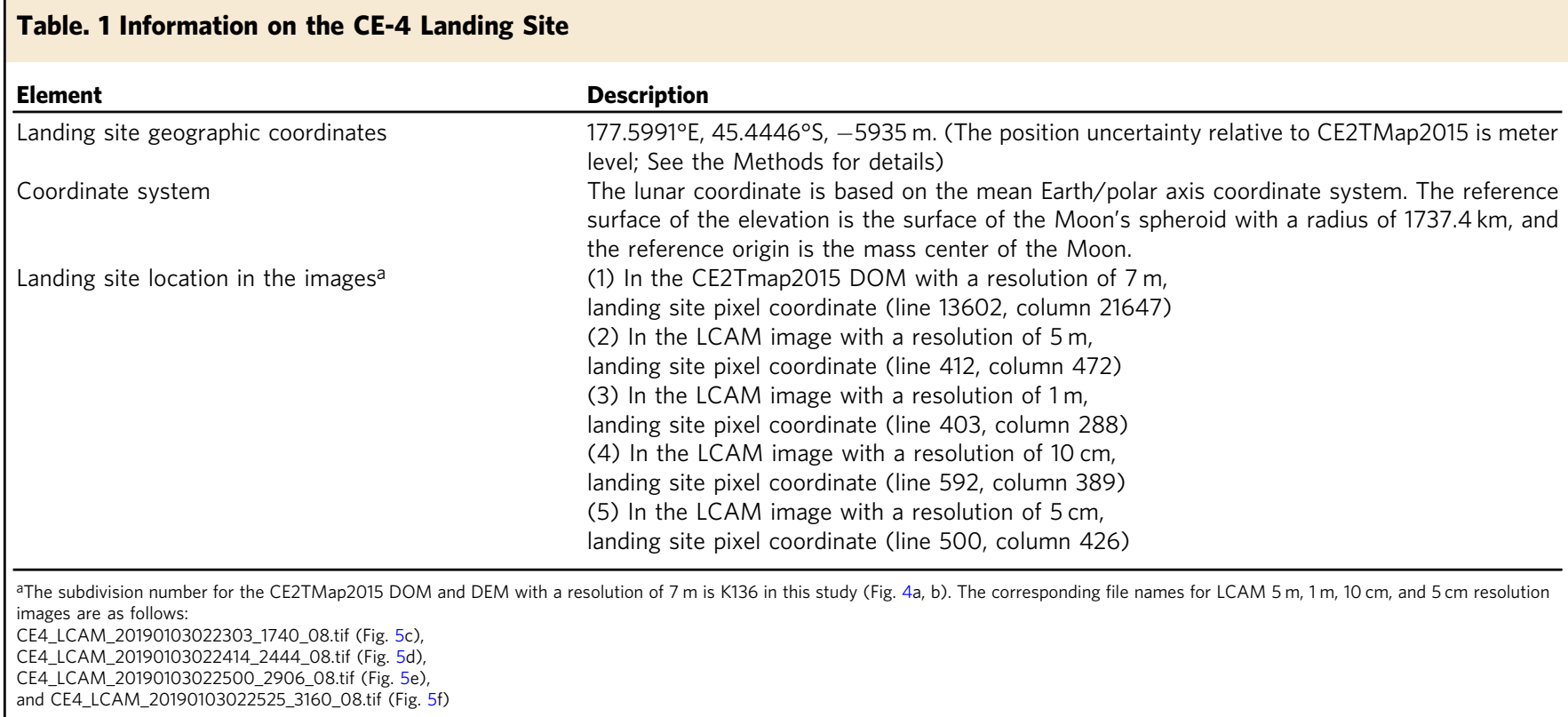

In contrast, the landing site of $\mathrm{CE}-4$ is located southeast of the Von Kármán crater on the lunar farside. The length of the CE-4 nadir flight trace is also $450 \mathrm{~km}$ from north to south. However, the terrain of the nadir flight trace shows a large variation with a maximum variation of $6000 \mathrm{~m}$ (Fig. 6). Because the pointing position of the ranging beam is different from the final landing site and the terrain variation is obvious, if ranging measurements were introduced for a correction in advance, there would be a safety risk. Under its trace control strategy, CE-4 performed rapid attitude adjustments at an altitude of $8 \sim 6 \mathrm{~km}$, and ranging measurements were introduced to correct the IMU bias and drift. This strategy ensured a safe landing of CE-4. The recovered trajectory showed that the lander attitude was adjusted to enable thrusting for near-vertical descent at an altitude of $5635 \mathrm{~m}$. Thus, the powered descent trajectory of CE-4 is obviously different from that of CE3 (Fig. 7).

Based on the sequence images of the LCAM, we reconstructed the powered descent trajectory of the CE-4 lander through photogrammetry. Combining the generated $\mathrm{cm}$-resolution terrain data near the landing site with the NCAM stereoscopic images, the geographical location of the landing site was calculated in this study. The horizontal and the vertical RMS errors of the landing site are $0.7 \mathrm{~m}(1 \delta)$ and $1.0 \mathrm{~m}(1 \delta)$ respectively (see the Methods section for details). 
a

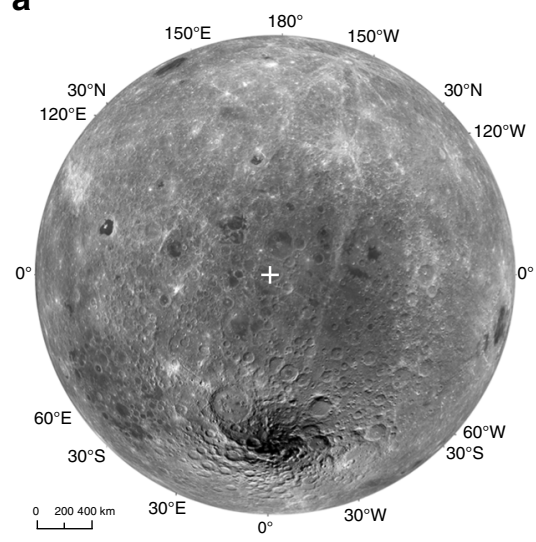

b

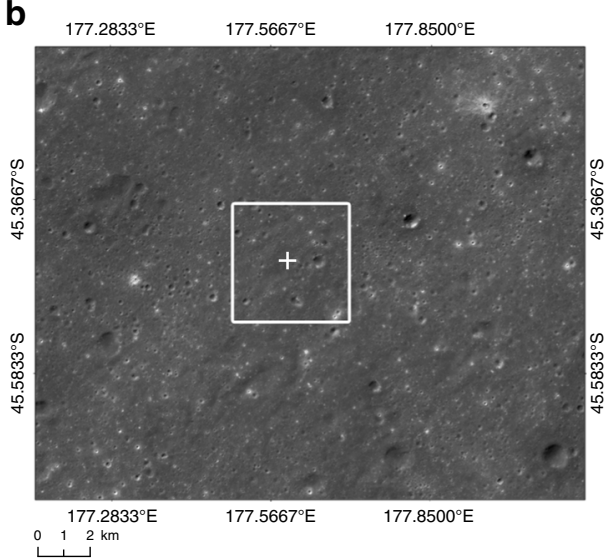

C

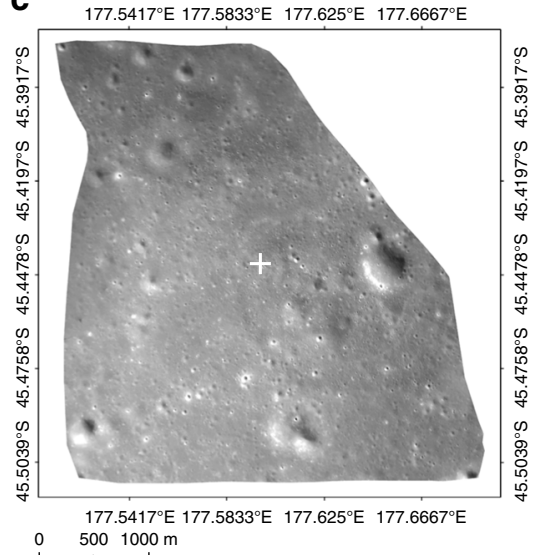

d

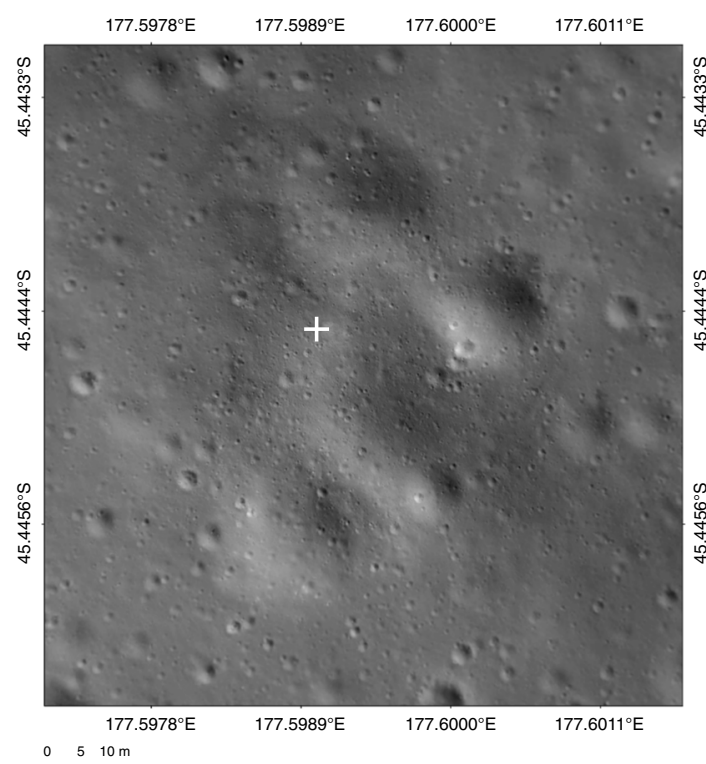

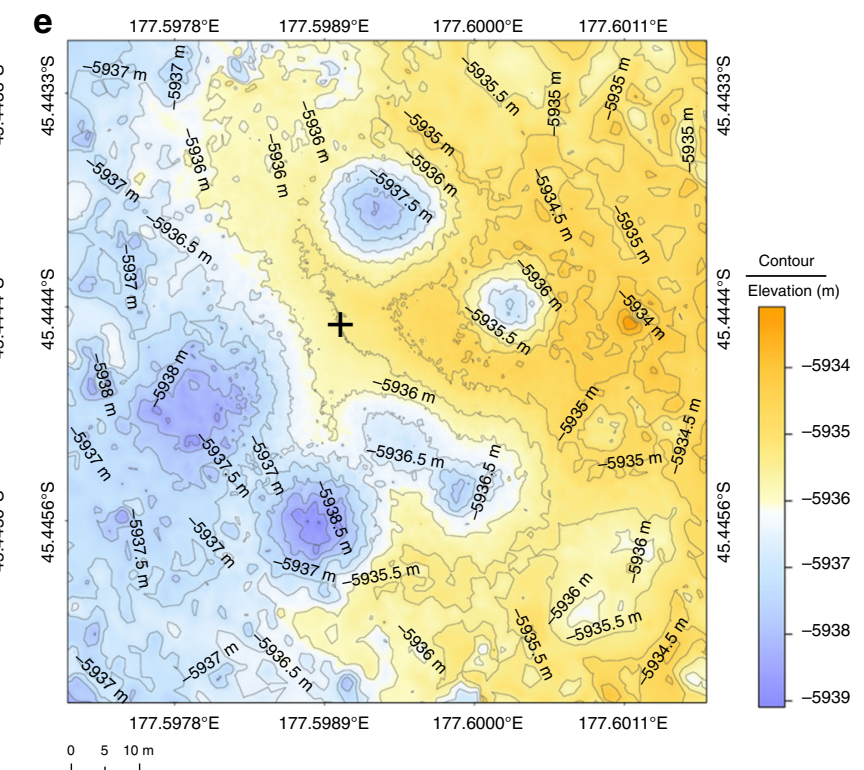

$5 \quad 5 \quad 10 \mathrm{~m}$

Fig. 3 Location of the CE-4 landing site. The + is the identified landing location. $\mathbf{a}, \mathbf{b}$ are the CE-2 DOMs, using the CE-4 landing site as the projection center of the azimuth projection. $\mathbf{c}$ is the LCAM DOM with a resolution of $5 \mathrm{~m} / \mathrm{p}$. $\mathbf{d}$ is the LCAM DOM, which is generated by the LCAM images with resolutions from $21.4 \mathrm{~cm} / \mathrm{p}$ to $0.2 \mathrm{~cm} / \mathrm{p}$ and is uniformly resampled at $5 \mathrm{~cm} / \mathrm{p}$ during mapping. $\mathbf{e}$ is a shaded-relief map with contour line produced by the LCAM DEM, which area and resolution are the same as in (d)

Compared with the positioning results of the landing site based on LRO terrain data $\left(177.5885^{\circ} \mathrm{E}, 45.4561^{\circ} \mathrm{S},-5927 \mathrm{~m}\right)^{24}$, our results show a $226 \mathrm{~m}$ deviation along the latitude direction and a $348 \mathrm{~m}$ deviation along the longitude direction. The total positional deviation is $415 \mathrm{~m}$, which reflects the deviation of the two sets of terrain data on the lunar farside (see the Methods section for details). A feasible method to effectively eliminate this deviation is to establish absolute control points on the lunar farside.

As a permanent artificial landmark on the lunar farside, the location of the CE-4 lander was precisely confirmed using CE-2 and CE- 4 images and can serve as a potential control point on the farside. The result will provide a worthy geodetic data point for studies on lunar control points, high-precision lunar mapping, and subsequent lunar exploration, such as for the Yutu-2 rover.

\section{Methods}

Instruments and dataset descriptions. The dataset used in this study includes landing camera (LCAM) images, navigation camera (NCAM) images and the Chang'E-2 (CE-2) global lunar terrain (CE2TMap2015) 20,21 .

The LCAM is one of the scientific payloads installed on the bottom of the Chang'E-4 (CE-4) lander. According to the analysis of the characteristics of the

\section{Table 2 The performance parameters of the LCAM}

\begin{tabular}{lll} 
No. & Name & Performance parameters \\
\hline 1. & Wavelength range $(\mathrm{nm})$ & $419 \sim 77$ \\
2. & Field of view $\left({ }^{\circ}\right)$ & $45.3^{\circ} \times 45.3^{\circ}$ \\
3. & Focal Length $(\mathrm{mm})$ & 8.5 \\
4. & Effective pixel numbers & $1024 \times 1024$ \\
5. & Pixel size on Focal plane $(\mu \mathrm{m})$ & 6.7 \\
6. & Frame rate (fps) & 10 \\
7. & Quantized value (bit) & 8 \\
8. & Data compression ratio & $8: 1$ \\
\hline
\end{tabular}

LCAM sequence images, the CE-4 lander gradually approached the landing area using a vertical descent mode after attitude adjusting phase. The overlap of the LCAM sequence images exceeds $94 \%$. Over $40 \%$ of the images cover the landing site. The rotation and translation between adjacent images are slow. Therefore, 180 images in $1 \mathrm{~s}$ intervals during this phase were selected for the reconstruction of the powered descent trajectory and landing area topography. The main performance parameters of the LCAM are shown in Table 2.

The NCAM is an engineering payload onboard the CE-4 rover and is installed on the top of the rover mast. It consists of two optical systems. The NCAM obtained images surrounding the landing site at the top of the lander before the 
a

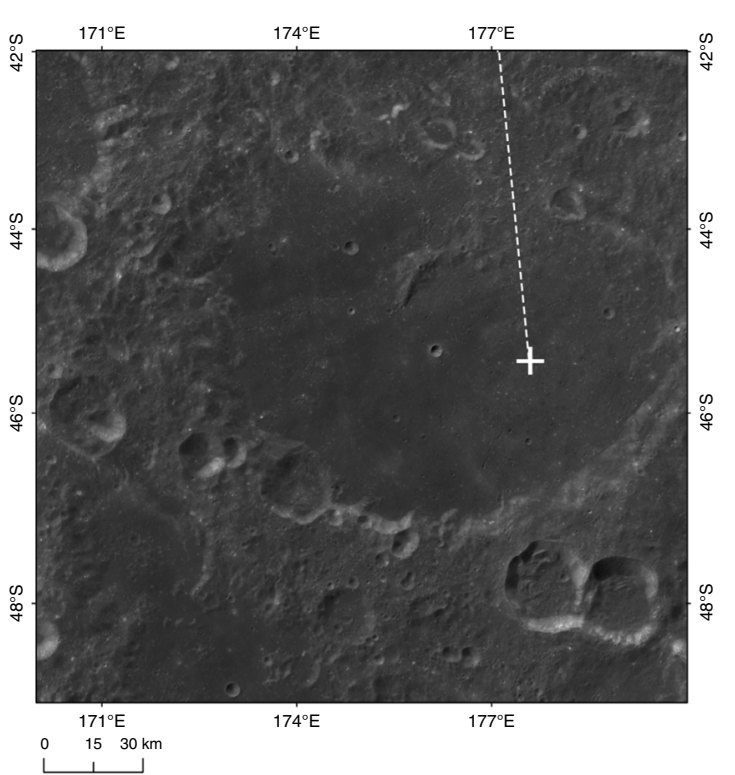

b

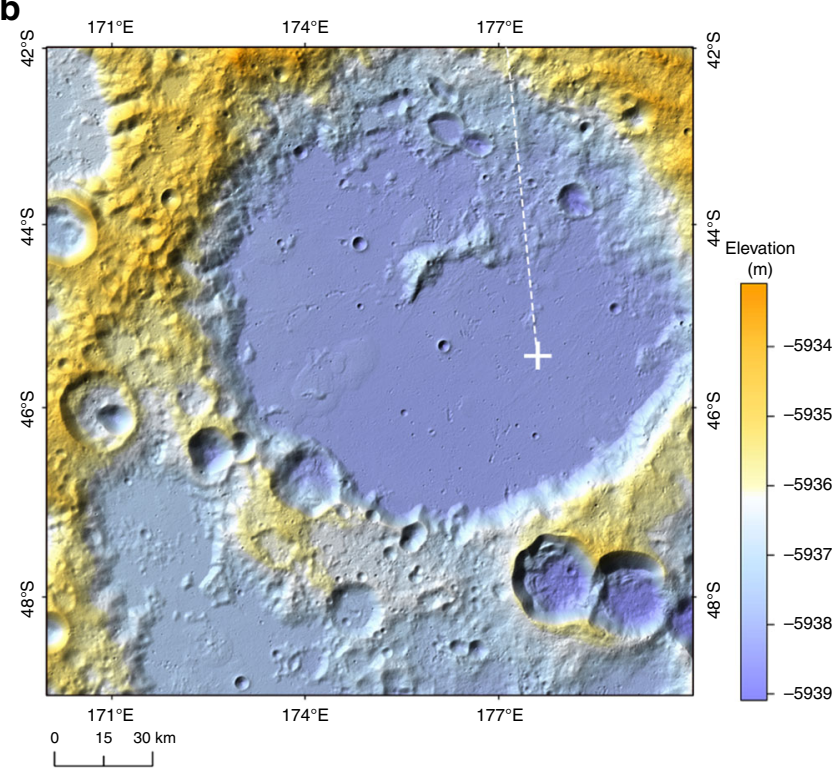

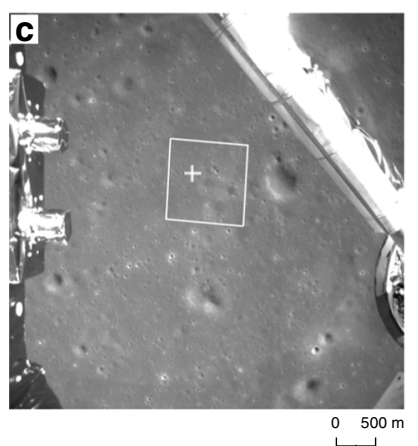
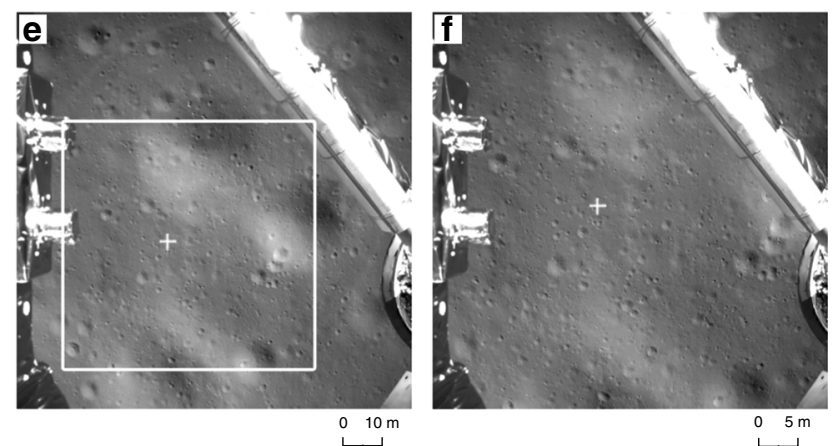

Fig. 4 The CE-4 landing site on CE2TMap2015 and CE-4 LCAM images. The + is the identified landing location. a Landing site on the Chang'E-2 DOM. b Landing site on a shaded-relief map made by the Chang'E-2 DEM. The projection is the positive-axis isometric Mercator projection, which has a standard parallel at $45^{\circ} \mathrm{S}$. White dotted line represents the approach trajectory of CE-4; c-e, $f$ are the LCAM images with spatial resolutions of $5 \mathrm{~m} / \mathrm{p}$, $1 \mathrm{~m} / \mathrm{p}, 10 \mathrm{~cm} / \mathrm{p}$, and $5 \mathrm{~cm} / \mathrm{p}$, respectively. The white rectangles in (c-e) represent the borders of (d-f), respectively

rover separated from the lander. Based on these images, topographic data of the landing site with $\mathrm{cm}$-level accuracy can be reconstructed by close-range photogrammetry. Debris scattered near the landing site can be accurately identified. Because the relationship between the location of the NCAM and the CE-4 lander is known, the precise position of the landing site can be located by combining the images of the LCAM and $\mathrm{NCAM}^{25}$. The main performance parameters of the NCAM are shown in Table 3.

The georeferenced data used in this study are CE2TMap2015, which include the DOM and DEM with resolutions of $7 \mathrm{~m} / \mathrm{p}$ and $20 \mathrm{~m} / \mathrm{p}$, respectively. These data are produced using CE-2 stereo images with a resolution of $7 \mathrm{~m}$. The average horizontal and elevation relative position errors are $5 \mathrm{~m}$ and $2 \mathrm{~m}$, respectively. Compared to the absolute positions of 5 laser reflectors located on the Moon, the horizontal positional deviation of CE2TMap2015 is $21 \sim 97 \mathrm{~m}$, and the elevation deviation is $2 \sim 19 \mathrm{~m}^{20,21}$.

LRO terrain data is used for CE-4 landing site position comparison. Orbit overlap analyses show that, LRO spacecraft ephemeris can be improved to less than $10 \mathrm{~m}$ horizontally and $1 \mathrm{~m}$ vertically by combining radiometric tracking data and LOLA data with the GRAIL gravity model ${ }^{25-27}$. As a result, the LRO terrain data uncertainty is about 20 meters $^{28}$ and the positional deviation from the 5 laser reflectors is $<5.2 \mathrm{~m}^{29}$. A pair of LRO NAC observations of the CE-4 landing site were collected on 1 February 2019 (M1303619844LR, M1303640934LR), and were used to create a digital terrain model (DTM) on the LOLA-plus-GRAIL coordinate framework. The coordinates of CE-4 lander were derived $\left(177.5885^{\circ} \mathrm{E}, 45.4561^{\circ} \mathrm{S}\right.$, $-5927 \mathrm{~m})^{24}$ based on the DTM.

It can be seen that both CE2TMap2015 and LRO terrain data show good internal consistency. However, the positioning deviation of the two sets of terrain data on the lunar farside will both increase due to several reasons, such as orbital measurement error, the lumpy gravity field of the Moon on the lunar farside, small uncertainties in camera model (distortion, FL), etc. Compared to the LRO terrain data, the average global positional deviation of CE2TMap2015 is $354 \mathrm{~m}$ with a standard deviation of $228 \mathrm{~m}(1 \delta)^{20,21,30}$, which mainly results from large deviation

\begin{tabular}{|lll|}
\hline \multicolumn{2}{|l|}{ Table 3 The performance parameters of the NCAM } \\
No. & Name & Performance parameters \\
\hline 1. & Focal Length $(\mathrm{mm})$ & Left camera: 17.69 \\
& & Right camera: 17.98 \\
2. & Effective pixel numbers & $1024 \times 1024$ \\
3. & Pixel size on Focal plane $(\mu \mathrm{m})$ & 15 \\
4. & Baseline length $(\mathrm{mm})$ & 269.69 \\
5. & Quantized value (bit) & 8 \\
\hline
\end{tabular}

of two sets of terrain data on the farside. The $415 \mathrm{~m}$ discrepancy between our coordinates and the LRO terrain data coordinates for the CE- 4 lander is within the positional deviation range.

Technical flowchart. A total of 180 images (one image/per second) from the LCAM sequence images and 18 pairs of NCAM stereo images captured at the top of the CE4 lander were selected for this work. Figure 8 shows the technical flowchart of the trajectory recovery and landing site position determination.

CE-4 lander descent trajectory reconstruction. First, evenly distributed points (or tie points) were automatically extracted using scale-invariant feature transform (SIFT) feature matching ${ }^{31}$. Then, least squares matching was applied to these tie points to achieve more precise matching results, and a random sample consensus (RANSAC)-based optimization algorithm was used to automatically eliminate mismatches 32,33 . A total of 30992 tie points, evenly distributed throughout the 
a

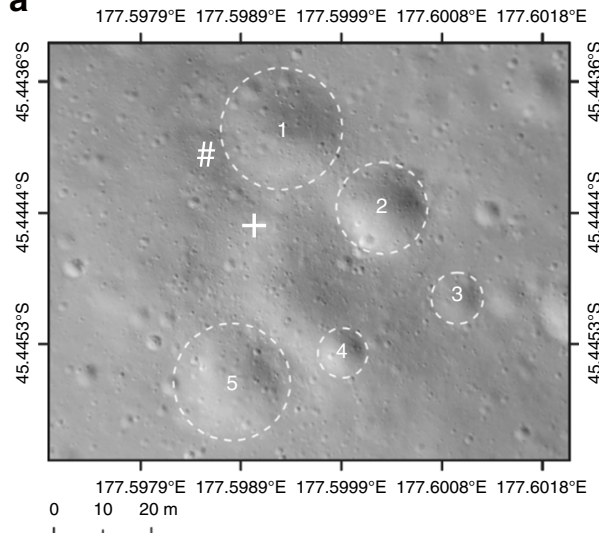

b

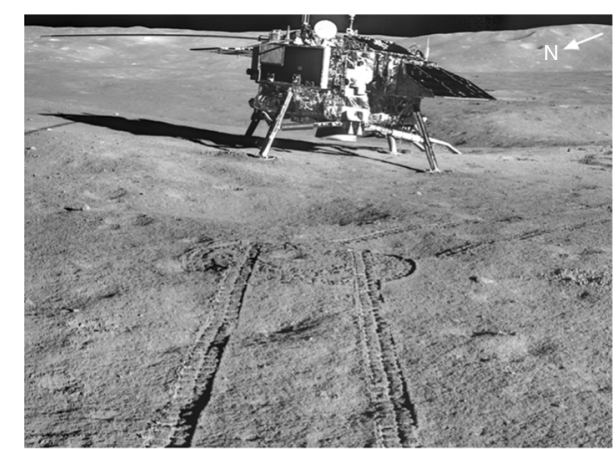

c

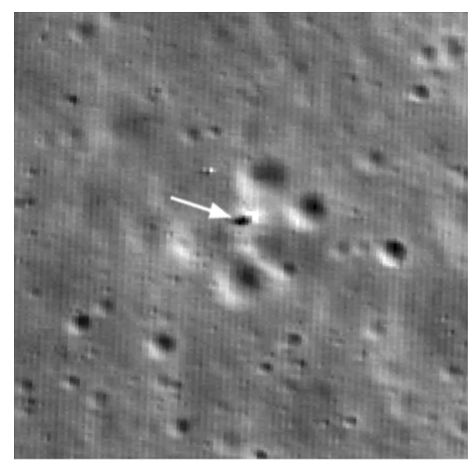

d

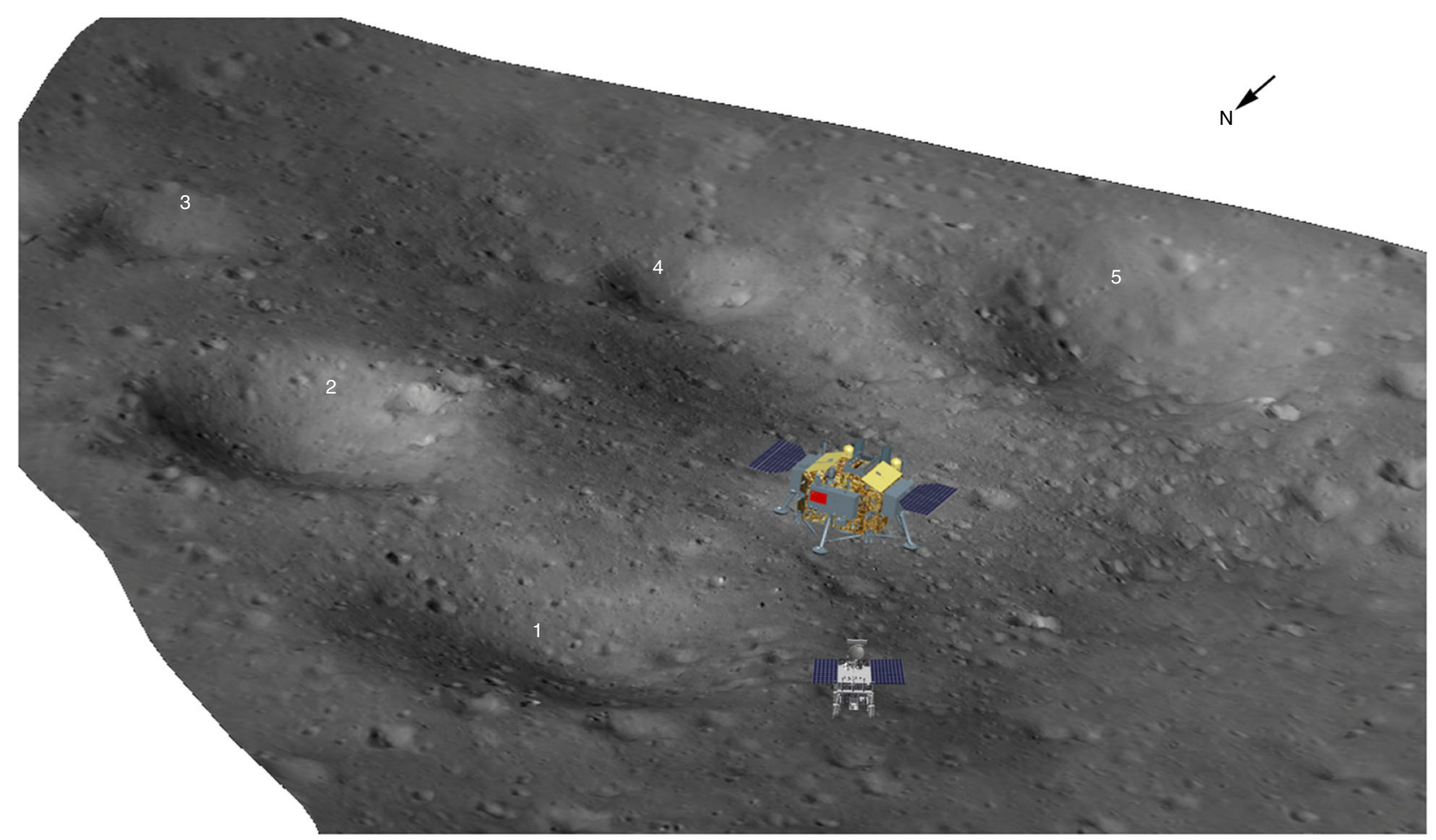

Fig. 5 Position of the CE-4 landing site. a The CE-4 landing site on the $5 \mathrm{~cm} / \mathrm{p}$ resolution LCAM DOM, in which the + is the identified landing location and the \# is the location of Yutu-2 on 12 January 2019. Five prominent craters around the landing site are marked. (b) CE-4 lander image obtained from the northwest of the landing site by the Yutu-2 PCAM at the location labeled in (a). (c) LRO NAC image of the CE-4 landing site collected on 1 February 2019 (M1303640934LR), in which the arrow indicates the location of CE-4 landing site. (d) Three-dimensional landscape map of the landing site generated by the DEM and DOM data through reconstruction of CE-4 LCAM sequential images acquired within $100 \mathrm{~m}$ above the lunar surface, in which the location of CE-4 lander and Yutu-2 is the same as labeled in (a). While landing position was determined from LCAM orbital data (a), the orientation of the lander was determined using PCAM image taken from the ground (b). Prominent craters are also marked in (d), as in (a)

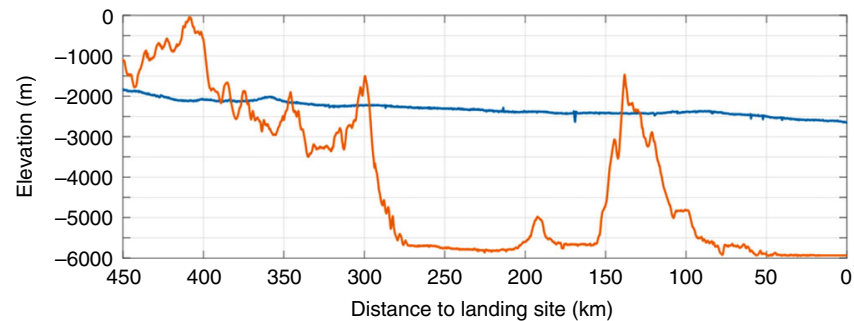

Fig. 6 Elevation variations of the lunar terrain along the CE-3 and CE-4 nadir flight traces. The blue and orange lines represent the elevation variation for the CE-3 and CE-4 nadir flight trace, respectively. Elevation is given with respect to the Moon's spheroid with a radius of $1737.4 \mathrm{~km}$ overlaps of the LCAM images, were extracted. The relative positional relationship of these images can be precisely restored according to these tie points.

Facilitated by one LRO NAC image (M178833263LC), 13 evenly distributed GCPs close to the landing site (including 12 horizontal and vertical GCPs and 1 vertical GCP) were extracted from the CE2TMap2015 map and LCAM images. The centers of some easily recognized small craters were mainly selected to ensure the selection accuracy of the GCPs.

According to photogrammetric bundle adjustment theory ${ }^{22,23}$, the relationship between the lunar surface points, the corresponding image points and the camera projection center can be expressed by the classical collinear equation, which is as follows.

$$
\begin{aligned}
& x-\mathrm{x}_{0}+\Delta x=-f \frac{r_{11}\left(X-X_{s}\right)+r_{21}\left(Y-Y_{s}\right)+r_{31}\left(Z-Z_{s}\right)}{r_{13}\left(X-X_{s}\right)+r_{23}\left(Y-Y_{s}\right)+r_{33}\left(Z-Z_{s}\right)} \\
& y-y_{0}+\Delta y=-f \frac{r_{12}\left(X-X_{s}\right)+r_{22}\left(Y-Y_{s}\right)+r_{32}\left(Z-Z_{s}\right)}{r_{13}\left(X-X_{s}\right)+r_{23}\left(Y-Y_{s}\right)+r_{33}\left(Z-Z_{s}\right)}
\end{aligned}
$$

where $(x, y)$ are the image coordinates of the feature points, $(x 0, y 0)$ are the image coordinates of the image principal point, $(\Delta x, \Delta y)$ are self-calibration correction 
a

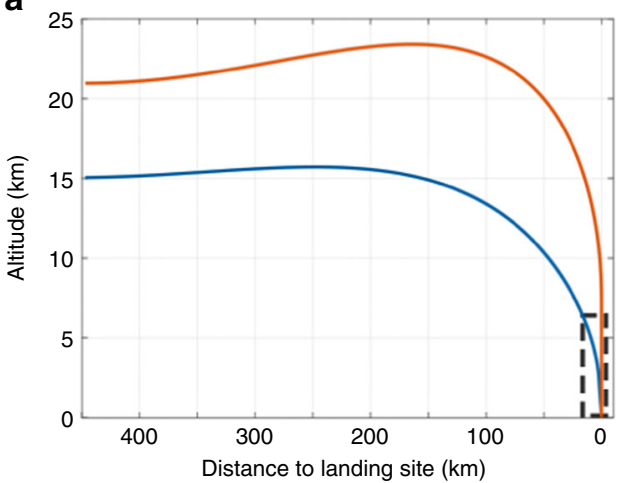

b

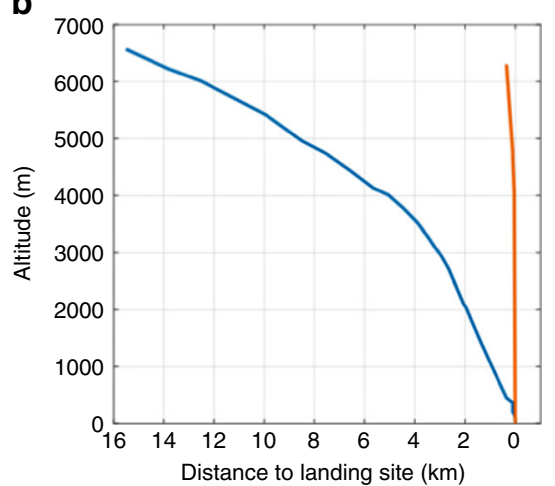

Fig. 7 Comparison of powered descent trajectory between the CE-3 and CE-4. The blue and orange lines represent trajectories of the CE-3 and CE-4, respectively. a The designed trajectories of the entire powered descent, in which the marked rectangle corresponds to designed trajectories below the altitude of $\sim 6 \mathrm{~km}$; $\mathbf{b}$ Magnified portion of the reconstructed trajectory below the altitude of $\sim 6 \mathrm{~km}$. Altitude is given with respect to final landing site level

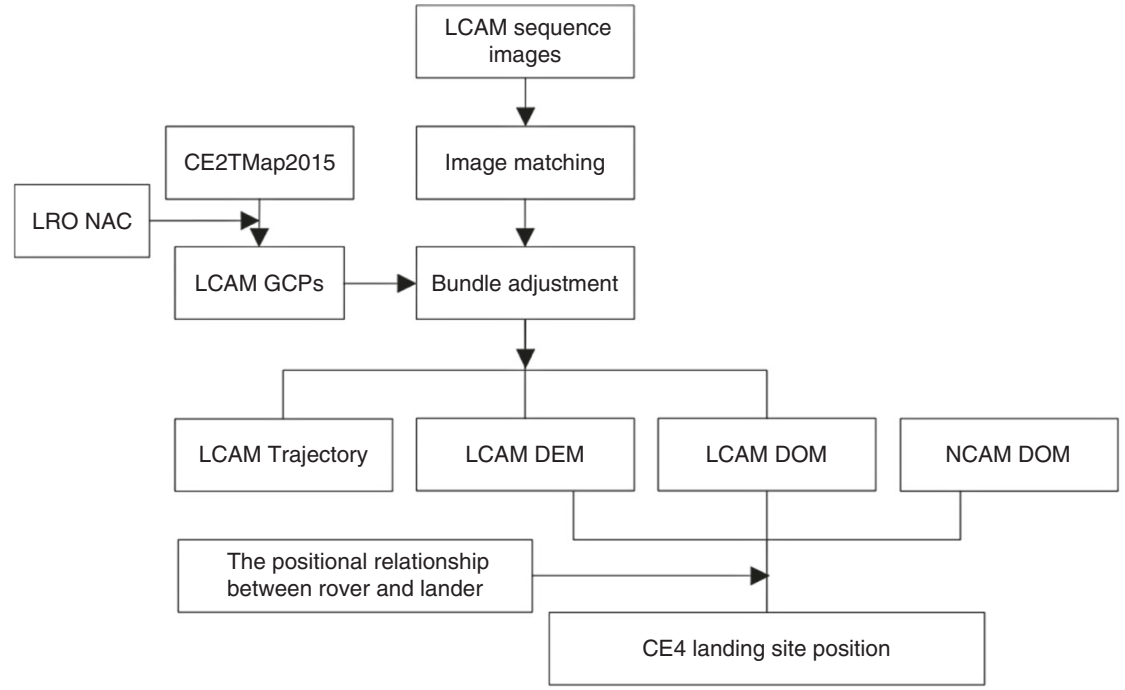

Fig. 8 Data processing flowchart

terms of the camera, including lens distortion correction, $(X, Y, Z)$ are the lunar space coordinates of the feature points, $\left(X_{s}, Y_{s}, Z_{s}\right)$ are the lunar space coordinates of the image principal point, $f$ is the focal length of the camera, and $r 11-r 33$ are the elements of the rotation matrix $\mathrm{R}(\varphi, \omega, \kappa)$, which is formed by the Euler attitude angle $(\varphi, \omega, \kappa)$ of the LCAM and used to translate feature points from the lunar space coordinates to the image coordinates. $\left(X_{s}, Y_{s}, Z_{s}, \varphi, \omega, \kappa\right)$ forms the exterior orientation (EO) parameters of the LCAM. Using Taylor's formula, the linearized error equations of each pixel can be derived by formula (1). The EO parameters of each image and the lunar space coordinates of each feature point can be solved by the least squares method. We implemented the photogrammetric bundle adjustment theory by ContextCapture Master software in this study. Therefore, we can use the location of each image to reconstruct the descent trajectory of the CE4 lander.

After the adjustment, the RMS error of the reprojection error for all the tie points is 0.5 pixels. The horizontal and vertical RMS errors of the GCPs are, respectively, $0.715 \mathrm{~m}(1 \delta)$ and $1.040 \mathrm{~m}(1 \delta)$ (Table 4), which represent the accuracy of the CE- 4 descent trajectory reconstruction.

Precise localization of the landing site. First, the position and attitude of the LCAM images were obtained by the landing trajectory reconstruction. Then, a pixel-level point cloud was generated by the image dense matching technique ${ }^{34,35}$, and a DEM of the LCAM with a $5 \mathrm{~cm}$ spatial resolution within $80 \mathrm{~m}$ around the landing site was interpolated from the triangulated irregular network (TIN). Finally, the same resolution and coverage DOM of the LCAM was produced using the image reprojection technique with the DEM and EO parameters.

Similarly, we produced the DEM and DOM of the NCAM with a $2 \mathrm{~cm}$ spatial resolution within $30 \mathrm{~m}$ around the landing site using the NCAM images. The only difference for this process is that the GCPs were selected from the LCAM terrain data. Considering that the spatial resolution of the NCAM image is close to that of the descent camera terrain data, some small stones scattered around the landing

\section{Table 4 Horizontal and vertical deviations of the GCPs for} the LCAM

\begin{tabular}{lllc} 
Name & $\begin{array}{l}\text { RMS of reprojection } \\
\text { error (pixel) }\end{array}$ & $\begin{array}{l}\text { Horizontal } \\
\text { error }(\mathbf{m})\end{array}$ & $\begin{array}{c}\text { Vertical } \\
\text { error }(\mathbf{m})\end{array}$ \\
\hline LGCP01 & 0.35 & 1.522 & -2.319 \\
LGCP02 & 0.74 & 2.560 & 0.504 \\
LGCP03 & 0.21 & 1.327 & -1.528 \\
LGCP04 & 0.72 & 2.933 & 1.365 \\
LGCP05 & 0.55 & 0.897 & 0.807 \\
LGCP06 & 0.67 & 1.565 & -0.642 \\
LGCP07 & 0.72 & 1.685 & -0.513 \\
LGCP08 & 0.23 & 0.710 & 0.610 \\
LGCP09 & 0.93 & 0.795 & 0.799 \\
LGCP10 & 1.08 & 1.048 & -0.788 \\
LGCP11 & 0.75 & 0.855 & 0.420 \\
LGCP12 & 0.84 & 0.865 & -0.483 \\
LGCP13 & 0.11 & $/$ & 0.065 \\
Global RMS & 0.61 & 0.715 & 1.040 \\
\hline
\end{tabular}

site which can be easily identified were selected as GCPs to ensure the accuracy of the GCP selection (Fig. 9). A total of 14 GCPs were selected.

A total of 21,091 tie points were extracted using the image matching method. The RMS error of the reprojection error for all tie points is better than 0.2 pixels after the adjustment. The GCP horizontal RMS error is $0.017 \mathrm{~m}(1 \delta)$, and the vertical RMS 
a

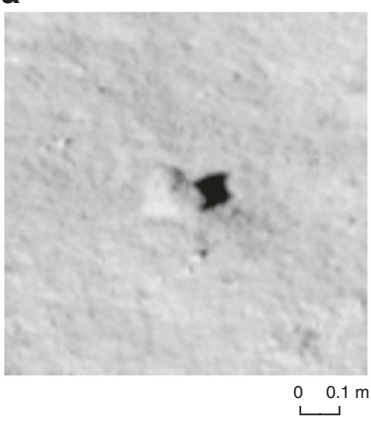

b

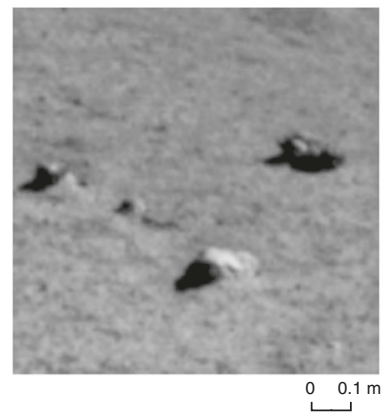

c

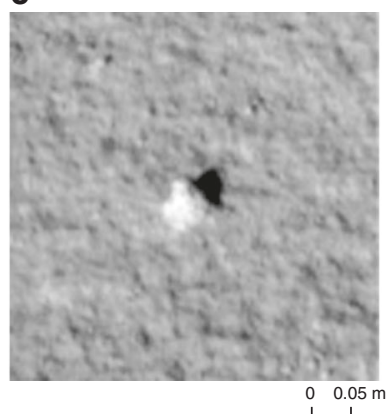

d

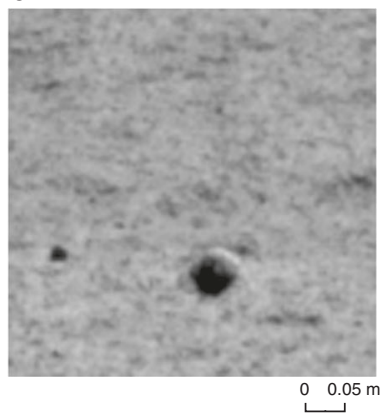

Fig. 9 Small rocks used as GCPs. a-d are stones in the NCAM image that can also be easily identified in the LCAM DOM

\section{Table 5 Horizontal and vertical deviations of the GCPs for the NCAM}

\begin{tabular}{lllr} 
Name & $\begin{array}{l}\text { RMS of the } \\
\text { reprojection } \\
\text { error (pixel) }\end{array}$ & $\begin{array}{l}\text { Horizontal } \\
\text { error (m) }\end{array}$ & $\begin{array}{l}\text { Vertical } \\
\text { error (m) }\end{array}$ \\
\hline NGCP01 & 0.077 & 0.053 & -0.038 \\
NGCP02 & 0.006 & 0.034 & 0.038 \\
NGCP03 & 0.007 & 0.014 & -0.037 \\
NGCP04 & 0.010 & 0.032 & 0.048 \\
NGCP05 & 0.005 & 0.021 & 0.017 \\
NGCP06 & 0.007 & 0.028 & -0.065 \\
NGCP07 & 0.003 & 0.027 & -0.004 \\
NGCP08 & 0.007 & 0.040 & -0.009 \\
NGCP09 & 0.013 & 0.075 & 0.013 \\
NGCP10 & 0.005 & 0.049 & -0.027 \\
NGCP11 & 0.005 & 0.031 & 0.014 \\
NGCP12 & 0.005 & 0.020 & 0.001 \\
NGCP13 & 0.005 & 0.015 & 0.040 \\
NGCP14 & 0.017 & 0.014 & 0.015 \\
Global RMS & 0.012 & 0.017 & 0.031 \\
\hline
\end{tabular}

error is $0.031 \mathrm{~m}(1 \delta)$ (Table 5). According to the positional relationship between the NCAM and the origin of the lander's body coordinate system (LBCS), the horizontal position of the vertical projection of the LBCS origin in the LCAM terrain data is $177.5991^{\circ} \mathrm{E}, 45.4446^{\circ} \mathrm{S}$, and the elevation value is $-5935 \mathrm{~m}$.

\section{Data availability}

CE2TMap2015 and CE-4 images are available at the Data Publishing and Information Service System of China's Lunar Exploration Program (http://moon.bao.ac.cn/). Datasets generated and/or analyzed during the current study are available from the corresponding author.

Received: 23 January 2019 Accepted: 23 August 2019

Published online: 24 September 2019

\section{References}

1. Li, S., Jiang, X. Q. \& Tao, T. Guidance Summary and Assessment of the Chang'e3 Powered Descent and Landing. J. Spacecraft Rockets 53, 258-277 (2016).

2. Zhang, H. H. et al. Guidance navigation and control for Chang'E-3 powered descent (in Chinese). Sci. Sin. Tech. 44, 377-384 (2014).

3. Jia, Y. et al. Chang'E-3 system pinpoint landing localization based on descent image sequence (in Chinese). Chin. Sci. Bull. (Chin. Ver.). 59, 1838-1843 (2014).

4. Klumpp, A. R. Apollo Lunar Descent Guidance, R-695. (Charles Stark Draper Laboratory of Massachusetts Institute of Technology, Cambridge, 1971).

5. Li, F. et al. Influence analysis of terrain of the farside of the Moon on softlanding. J. Deep Space Exploration 4, 143-149 (2017).

6. Cao, J. F. et al. Determination of Impact Site of CE -1 Using USB and VLBI Data. J. Astronautics 31, 1724-1729 (2010).
7. Edwards, C. D. et al. Telecommunications relay support of the Mars Phoenix lander mission. IEEE Aerospace Conference. pp. 1-13 (2010).

8. Folkner, W. M. et al. Interior structure and seasonal mass redistribution of Mars from radio tracking of Mars Pathfinder. Science 278, 1749-1751 (1997).

9. Li, J. L. et al. Determination of the controlled landing trajectory of Chang'E1 satellite and the coordinate analysis of the landing point on the Moon. Chin. Sci. Bull. 55, 752-757 (2010).

10. Li, P. J. et al. Kinematic statistical method using in the orbit determination of CE-1. Prog. Astron. 28, 290-300 (2010).

11. Li, R. et al. Initial results of rover localization and topographic mapping for the 2003 Mars Exploration Rover mission. Photogrammetric Eng. Remote Sens. 71, 1129-1142 (2005)

12. Yoder, C. F. \& Standish, E. M. Martian precession and rotation from Viking lander range data. J. Geophys. Res. 102(E2), 4065-4080 (1997).

13. Lee, A. Y. et al. Preliminary design of the guidance, navigation, and control system of the altair lunar lander. Conference: AIAA Guidance, Navigation, and Control Conference, https://arc.aiaa.org/doi/10.2514/6.2010-7717 (2010).

14. Ansar, A. \& Cheng, Y. An analysis of spacecraft localization from descent image data for pinpoint landing on Mars and other cratered bodies. Photogramm. Eng. Remote Sens. 71, 1197-1204 (2005).

15. Liu, B. et al. Descending and landing trajectory recovery of Chang'e-3 lander using descent images. J. Remote Sens. 18, 981-987 (2014a).

16. Liu, J. J. et al. Positioning of the CE-1's impact site based on CCD image data photographed during the controlled impact on the Moon. Sci. China Earth Sci. 41, 1714-1720 (2011).

17. Liu, J. J. et al. Reconstructing the landing trajectory of the CE-3 lunar probe by using images from the landing camera. Res. Astron. Astrophys. 14, 1530-1542 (2014).

18. Matthies, L. et al. Computer vision on Mars. Int J. Comput Vis. 75, 67-92 (2007).

19. Xiong, Y., Olson, C. F. \& Matthies, H. Computing depth maps from descent images. Mach. Vis. Appl. 16, 139-147 (2005).

20. Ren, X., et al. A global adjustment method for photogrammetric processing of Chang'E-2 stereo images. IEEE Transactions on Geoscience and Remote Sensing. https://doi.org/10.1109/TGRS.2019.2908813 (2019).

21. Li, C. L. et al. Lunar global high precision terrain reconstruction based on Chang'e-2 stereo images. Geomat. Inf. Sci. Wuhan. Univ. 43, 486-495 (2018).

22. Wang, Z. Z. Principles of Photogrammetry [with Remote Sensing]. Press of Wuhan Technical University of Surveying and Mapping. (Publishing House of Surveying and Mapping, Beijing, 1990).

23. Brown, D. C. A solution to the general problem of multiple station analytical stereo- angulation. Air Force Missile Test Center Report No. 58-8, Patrick AFB, Florida. (1958).

24. Topographic Map of the Chang'e 4 Site. http://www.lroc.asu.edu/posts/1100. April 30 (2019).

25. Lemoine, F. G. et al. GRGM900C: A degree 900 lunar gravity model from GRAIL primary and extended mission data. Geophys. Res. Lett. 41, 3382-3389 (2014).

26. Mazarico, E. et al. Orbit determination of the lunar reconnaissance orbiter. J. Geod. 86, 193-207 (2012).

27. Mazarico, E., et al. Improved orbit determination of lunar orbiters with lunar and gravity fields obtained by the GRAIL mission. In: Proceedings of the 44th Lunar and Planetary Science Conference. The Wood- lands, TX, Abstract \#2414 (2013).

28. Speyerer, E. J. et al. Pre-flight and on-orbit geometric calibration of the Lunar Reconnaissance Orbiter Camera. Space Sci. Rev. 200, 357-392 (2016).

29. Wagner, R. V. et al. Coordinates of anthropogenic features on the Moon. Icarus 283, 92-103 (2017)

30. Yan, W. et al. Orbit optimization of Chang'E-2 by global adjustment using images of the moon. Adv. Space Res. 56, 2389-2401 (2015). 
31. Lowe, D. G. Distinctive image features from scale-invariant keypoints. Int. J. computer Vis. 60, 91-110 (2004).

32. Fischler, M. A. \& Bolles, R. C. Random sample consensus: a paradigm for model fitting with applications to image analysis and automated cartography. Commun. ACM 24, 381-395 (1981).

33. Zhang, L. Automatic digital surface model (DSM) generation from linear array images. Mitteilungen- Institut fur Geodasie und Photogrammetrie an der Eidgenossischen Technischen Hochschule Zurich. (2005)

34. Hirschmüller, H. Semi-global matching-motivation, developments and applications. Photogrammetric Week 11, 173-184 (2011).

35. Re, C., et al. Evaluation of area-based image matching applied to DTM generation with Hirise images. ISPRS Annals of the Photogrammetry, Remote Sensing and Spatial Information Sciences (2012).

\section{Acknowledgements}

This research was funded by the Chang'E-4 mission of Chinese Lunar Exploration Program (CLEP) and the National Natural Science Foundation of China (Grant No.11941002). We thank the team of the CE-4 Project for their successful work, especially the GRAS of CLEP for their valuable and efficient assistance with providing the data and data calibration.

\section{Author contributions}

C.L.L., J.J.L., X.R., and W.Y. designed the research and wrote the paper. J.J.L, X.R., and W.Y. performed the calculations. Z.H. and J.Y. are the CE-4 Spacecraft System team members and helped with the NCAM data processing. X.G.Z., W.L.C., X.Y.G, D.W.L., X.T., X.X.Z., and T.N. helped with the LCAM, PCAM, and NCAM data calibration, processing and mapping. H.B.Z., W.Z. Y.S., and W.B.W are the Ground Research and Application System (GRAS) team members of and helped with data retrieval, data preprocessing and instrument operations.

\section{Additional information}

Supplementary Information accompanies this paper at https://doi.org/10.1038/s41467019-12278-3.

Competing interests: The authors declare no competing interests.

Reprints and permission information is available online at http://npg.nature.com/ reprintsandpermissions/

Peer review information Nature Communications thanks the anonymous reviewers for their contribution to the peer review of this work. Peer reviewers are available.

Publisher's note Springer Nature remains neutral with regard to jurisdictional claims in published maps and institutional affiliations.

(c) (i) Open Access This article is licensed under a Creative Commons Attribution 4.0 International License, which permits use, sharing, adaptation, distribution and reproduction in any medium or format, as long as you give appropriate credit to the original author(s) and the source, provide a link to the Creative Commons license, and indicate if changes were made. The images or other third party material in this article are included in the article's Creative Commons license, unless indicated otherwise in a credit line to the material. If material is not included in the article's Creative Commons license and your intended use is not permitted by statutory regulation or exceeds the permitted use, you will need to obtain permission directly from the copyright holder. To view a copy of this license, visit http://creativecommons.org/ licenses/by/4.0/.

(C) The Author(s) 2019 